\section{Características clínicas e sociais determinantes para o idoso sair de casa}

\author{
Clinical characteristics and social determinants in a \\ sample of non-homebound elderly
}

\section{Características clínicas y sociales determinantes \\ para que el anciano salga de casa}

\author{
${ }^{1}$ Instituto de Geriatria e \\ Gerontologia, Pontifícia \\ Universidade Católica do Rio \\ Grande do Sul, Porto Alegre, \\ Brasil. \\ Correspondência \\ P. Morsch \\ Instituto de Geriatria e \\ Gerontologia, Pontifícia \\ Universidade Católica do Rio \\ Grande do Sul. \\ Av. Ipiranga 6690, 3o andar \\ Porto Alegre, RS \\ 90610-000, Brasil. \\ patriciamorsch@hotmail.com
}

\begin{abstract}
This study aimed to assess social and clinical factors associated with the fact that older adults ( $\geq 60$ years) go out of their homes. The study interviewed 5,898 older adults identified through home visits, randomly selected in 59 cities in the State of Rio Grande do Sul, Brazil. Multivariate logistic regression was used to assess the association between the outcome and independent variables. Factors associated with going out were being men, younger and married, presence of arthrosis, ease in performing specific activities, and good self-rated health. Heart disease was a negative factor for going out. Given the importance of social activity for quality of life and the World Health Organization policy for active aging, it is extremely important to consider clinical conditions that allow the older adults to remain active in the community. Studies like this can help to adjust public policies for the elderly, especially acting on modifiable clinical and functional conditions.
\end{abstract}

Aged; Geriatrics; Social Participation
Patricia Morsch 1

Gustavo Nunes Pereira 1

Joel Hirtz do Nascimento Navarro 1

Margarete Diprat Trevisan 1

Diene Gomes Colvara Lopes 1

Ângelo José Gonçalves Bós 1

\section{Resumo}

O estudo objetivou avaliar fatores sociais e de saúde envolvidos no fato de o idoso (60 anos ou mais) sair de casa. Foram entrevistados 5.898 idosos identificados por visita domiciliar, aleatoriamente selecionados em 59 cidades do Rio Grande de Sul, Brasil. A associação entre o desfecho e as variáveis independentes foi analisada de forma múltipla por meio de regressão logística. Fatores favorecedores à saída de idosos de casa: ser do sexo masculino, ser de faixa etária mais jovem, ser casado, ter artrose, realizar atividades específicas com facilidade e ter boa autopercepção de saúde. A presença de cardiopatias foi um fator negativo para sair de casa. Em face da importância da vida social na qualidade de vida e na política de envelhecimento ativo da Organização Mundial da Saúde, é fundamental considerar condições clínicas que permitem aos idosos manterem-se ativos em comunidade. Estudos como este podem auxiliar na adequação das políticas públicas para idosos, principalmente atuando em condições modificáveis, como as clínicas e funcionais.

Idoso; Geriatria; Participação Social 


\section{Introdução}

O envelhecimento populacional é um fenômeno conhecido mundialmente. O Brasil destaca-se por viver esse processo de forma acelerada na última década 1. Entre 1980 e 2009, a expectativa de vida dos brasileiros aumentou mais de 10 anos, passando de 62,6 anos para 73,2 anos 2 . Além disso, estima-se que, em 2050, mais de 15\% da população brasileira terá 70 anos ou mais 2 .

A participação social e o desenvolvimento de habilidades pessoais são fundamentais para a manutenção da qualidade de vida nessa faixa etária ${ }^{3}$. Sociedades que proporcionam maior participação do idoso em atividades fora de sua residência têm maior capacidade de garantir sua autonomia e independência, estimulando o estabelecimento de novos contatos sociais e qualidade de vida 4 .

Contudo, as doenças crônicas, comuns ao envelhecimento, podem dificultar essa interação social do idoso. Um estudo apontou que $40,8 \%$ dos indivíduos idosos restritos às suas casas apresentavam duas a três comorbidades clínicas ou psiquiátricas e que $31,9 \%$ apresentavam quatro ou mais comorbidades 5 . Mesmo que este estudo não seja representativo de todos os idosos, ele sugere que, de uma maneira geral, aqueles que não saem de casa apresentam mais comorbidades. Esses fatos podem explicar a menor autopercepção de saúde encontrada em idosos restritos à sua residência 6 .

Embora a literatura, principalmente internacional, sugira a existência de alguns fatores que favorecem à permanência demasiada do idoso em sua residência, é importante realizar análise mais aprofundada sobre os principais aspectos de saúde que podem estar relacionados a essa questão, uma vez que essa situação tende a gerar um quadro de limitações físicas, sociais e emocionais, impedindo o envelhecimento ativo. Portanto, esta pesquisa objetivou avaliar quais fatores sociais e de saúde são determinantes no fato de o idoso sair de casa, de forma regular.

\section{Métodos}

Este estudo é uma análise secundária do banco de dados do estudo denominado Perfil dos Idosos do RS (PIRS), realizado pelo Instituto de Geriatria e Gerontologia da Pontifícia Universidade Católica do Rio Grande do Sul (IGG-PUCRS) em parceria com a Escola de Saúde Pública do Rio Grande do Sul (ESP/RS). O PIRS foi realizado no período de 2010 a 2011, em 59 cidades do Estado do Rio Grande do Sul, Brasil, e objetivou traçar um perfil dos idosos do estado, inspirado no Guia Global: Cidade Amiga do Idoso, publicado em 2008 pela Organização Mundial da Saúde (OMS) 7. Os métodos adotados no PIRS basearam-se na metodologia da pesquisa Idosos do Rio Grande do Sul 8, na qual cidades com mais de 25 mil habitantes foram aleatoriamente selecionadas. Os setores censitários de cada cidade receberam numeração sequencial com sorteio dos números. O número de setores sorteados por município foi relacionado ao número de idosos estimados, utilizando-se a mesma proporção de $3 \%$ empregada na pesquisa anterior. Foram entrevistados oito idosos por setor, sequencialmente um idoso do sexo masculino e uma idosa do sexo feminino. Dessa forma, foi estabelecido um "pulo" de oito domicílios: após a identificação e entrevista de um idoso em um domicílio, reiniciava-se a identificação de novo participante a partir da oitava residência.

O PIRS caracterizou-se, assim, por ser descritivo, populacional, de base domiciliar, de corte transversal, com coleta aleatória mediante entrevista face a face. A população estudada foi composta por idosos, com idade acima de 60 anos, de ambos os sexos e residentes em domicílios urbanos e rurais no Estado do Rio Grande do Sul. Nos casos de idosos com demência, foram entrevistados os cuidadores ou familiares. Para verificar a habilidade cognitiva dos entrevistados, foi aplicado o teste de recordação de três palavras, tendo sido solicitado aos idosos que prestassem atenção nas palavras "irmão, chave e avião". O idoso deveria lembrar ao menos duas palavras. A metodologia utilizada no PIRS, bem como a descrição detalhada do recrutamento dos participantes, foi publicada recentemente pelos autores 9 .

No presente estudo, foram excluídos indivíduos que apresentavam déficit cognitivo ( $n=341)$, para reduzir o viés de resposta, assim como idosos acamados e que utilizavam algum dispositivo para a deambulação ou cadeira de rodas $(n=1.076)$, conforme ilustrado na Figura 1. Acreditamos que esses indivíduos apresentem outras barreiras para sair de casa, especialmente derivadas do ambiente, as quais não são contempladas nesta investigação.

O desfecho utilizado foi o fato de o idoso sair de casa. O questionamento foi feito por meio da seguinte pergunta: "Com que frequência costuma sair de sua casa ou propriedade?”. Criou-se, assim, a variável, contando se o idoso sai de casa ao menos uma vez na semana. As variáveis associadas foram fatores demográficos (idade em faixa etária, sexo e estado civil) e fatores relacionados à saúde, representados pelas variáveis queda no último ano, autopercepção de saúde e comorbidades (cardiopatias, osteoporose 
Figura 1

Fluxograma do estudo.

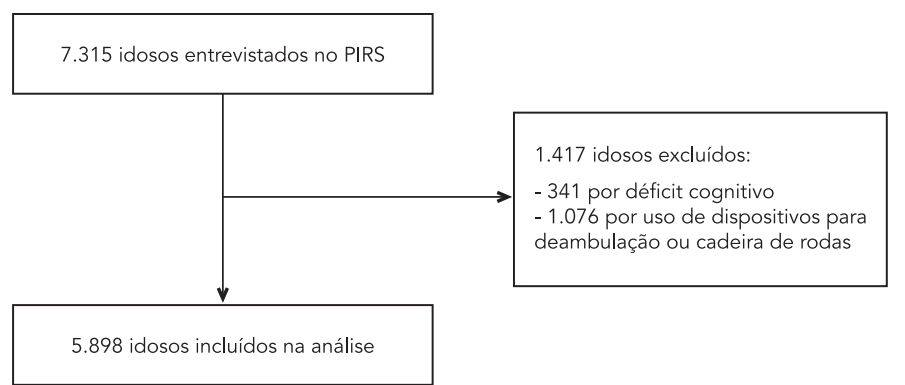

PIRS: Perfil dos Idosos do RS.

artrose, incontinência urinária e desnutrição). Originalmente, a questão referente à autopercepção de saúde contemplava as cinco categorias usualmente utilizadas (ótima, boa, regular, má e péssima), porém, para uma melhor análise dos dados, as respostas foram recodificadas para três categorias: boa (ótima e boa), regular (regular) e ruim (regular e péssima). As comorbidades utilizadas nessa análise foram escolhidas por serem patologias reconhecidamente causadoras de limitação funcional dos idosos. Elas foram coletadas de uma lista para evocação na qual o paciente respondia se já havia sido diagnosticado com alguma das comorbidades mencionadas. Outras patologias que podem influenciar no fato de o idoso sair de casa, como as doenças respiratórias, não foram contempladas neste artigo, pois não fizeram parte da investigação inicial. Em relação à presença de incontinência urinária, manteve-se, na análise, a opção "não soube responder", referindo-se à presença ou não da comorbidade, uma vez que, muitas vezes, os idosos sentem-se intimidados e envergonhados a relatar esse problema, sendo assim menos reportado 10,11. Em outros casos de não resposta, "não soube responder" ou "não se aplica", os dados foram excluídos da análise.

Os escores de atividades específicas e de realização das atividades básicas de vida diária (ABVDs) também foram analisados considerando-se o fato de o idoso sair de casa. O cálculo se deu com base na facilidade autorreferida para a realização das ABVDs (mudar-se da cama para uma cadeira ou vice-versa, banhar-se, vestir-se, alimentar-se sozinho, usar o banheiro para as suas necessidades) e de atividades específicas que exigem maior capacidade funcional (ca- minhar 400 metros ou quatro quadras, subir dez degraus ou um lance de escadas, levantar ou carregar objetos de cinco quilos, levantar-se de uma cadeira sem usar as mãos, abaixar-se e levantar-se para pegar algum objeto no chão, levantar os braços acima da cabeça, agarrar objetos firmemente com as mãos). Em ambas as variáveis, cada pergunta relacionada ao tipo de tarefa desempenhada apresentava uma resposta em escala de Likert, variando de 0 a 5 , sendo 0 equivalente à incapacidade de realizar a atividade e 5, à grande facilidade para a realização das tarefas ( 1 = muito difícil; 2 = difícil; 3 = mais ou menos fácil; 4 = fácil; 5 = muito fácil). $\mathrm{O}$ escore máximo de facilidade em realizar as tarefas específicas foi 35, e o escore máximo de facilidade na realização das ABVDs foi 25. Sendo assim, os escores foram divididos em três níveis com pontos de corte estabelecidos a partir dos tercis encontrados nos escores finais de cada variável, sendo denominados como níveis baixo, médio e alto. Para os níveis básicos, os pontos de corte foram os seguintes: menor de 20 (baixo), 20 (médio) e acima de 20 (alto). Os pontos de corte do nível funcional foram menor de 22 (baixo), entre 22 até 28 (médio) e 28 ou mais (alto).

As frequências de todas as variáveis associadas ao desfecho foram realizadas e expressas em tabelas. A associação entre o desfecho e as variáveis independentes foi analisada de forma múltipla, por meio de regressão logística. Nessa análise incluímos somente as variáveis significativas na análise simples, calculando, dessa forma, a chance associada a sair de casa. Os modelos finais ajustados de regressão logística foram alcançados mediante a metodologia de retirada sistemática das variáveis menos significativas, a partir do 
modelo completo, que incluía todas as variáveis independentes do estudo. Foram consideradas significativas as variáveis com associação que obtiveram nível de significância menor do que 0,05 . Os dados foram analisados por meio de programa Epi Info 3.5.3 (Centers for Disease Control and Prevention, Atlanta, Estados Unidos).

O projeto de pesquisa foi aprovado pelo Comitê de Ética da ESP/RS (protocolo 481/09) e pelo Comitê de Ética em Pesquisa da PUCRS (09/04931), cumprindo integralmente os princípios éticos contidos na Declaração de Helsinki (2000), além do atendimento à legislação específica do Brasil. Os autores declaram não haver conflitos de interesses associados a esta pesquisa e seus resultados.

\section{Resultados}

No total, foram avaliados 5.898 indivíduos idosos, sendo a maioria do sexo feminino $(51,9 \%)$, casados $(49,7 \%)$ e na faixa etária entre 60 e 69 anos (56,3\%). Dos participantes, 91,9\% não relataram queda prévia no período de um ano e referiram a autopercepção de saúde como boa (73,4\%), refletindo o baixo número de comorbidades. Nota-se que a maioria dos idosos apresenta certa facilidade em realizar as ABVDs e as atividades específicas. Esses resultados estão descritos na Tabela 1.

No modelo de regressão logística inicial, análise simples, apenas a variável independente desnutrição não foi significativamente associada com o desfecho "sair de casa" semanalmente. As demais variáveis e seus valores estatísticos encontram-se na Tabela 2.

Os modelos finais ajustados de regressão logística foram alcançados por meio da metodologia de retirada sistemática das variáveis menos significativas, valendo-se do modelo completo, que incluía todas as variáveis independentes do estudo. Dessa maneira, para o desfecho, mantiveram-se significativas as variáveis da Tabela 3. Níveis não significativos da variável estado civil foram mantidos para preservar as comparações significativas observadas.

De acordo com os resultados encontrados no modelo final da análise, idosos do sexo masculino têm maior chance de sair de casa do que as mulheres $(\mathrm{OR}=1,46 ; \mathrm{p}<0,001)$. As chances de sair casa foram proporcionalmente maiores na comparação de idade por década de vida; idosos mais jovens tendem a sair mais de casa $(\mathrm{p}<$ 0,001). Em relação ao estado civil, idosos solteiros apresentam menor chance de sair de casa na comparação com idosos casados $(\mathrm{OR}=0,70$; $\mathrm{p}<0,001$ ).
Idosos que consideram sua saúde regular têm menor chance associada a sair de casa do que os idosos que referem ter saúde boa ( $O R=$ $0,71 ; \mathrm{p}<0,001)$. O mesmo ocorre com aqueles que consideram sua saúde ruim, os quais apresentam chance $38 \%$ menor de saírem de casa $(\mathrm{p}=0,0023)$.

Em relação às comorbidades avaliadas, percebemos que idosos com cardiopatias apresentam chance $33 \%$ menor de sair de casa, diferentemente daqueles com artrose, que tendem a sair mais de casa $(O R=1,68 ; p<0,001)$. Os idosos com maior nível de facilidade para realizar as atividades específicas apresentaram maiores chances de sair de casa.

\section{Discussão}

Nosso estudo evidenciou, na comparação por década de vida, que idosos mais jovens e do sexo masculino tendem a sair mais de casa. Esses resultados se mantiveram significativos mesmo após a análise ajustada. Acreditamos que esse achado esteja relacionado ao fato de esses grupos apresentarem também maior grau de independência funcional e melhor avaliação da sua condição de saúde ${ }^{12}$. Um estudo de base populacional realizado em idosos do Sul do Brasil concluiu que aqueles mais longevos e do sexo feminino tendem a apresentar maior grau de dependência e maior número de doenças crônicas, o que aumentaria a dificuldade em sair do domicílio 13 . Outra pesquisa realizada em idosos de Porto Alegre, visando a observar o perfil sociodemográfico e as condições de saúde autorreferidas, também encontrou resultados semelhantes 14. Estudo realizado com idosos brasileiros de baixa renda igualmente relata que indivíduos mais jovens e do sexo masculino têm maior facilidade em realizar atividades fora de casa, como pegar ônibus e fazer compras 15. A respeito deste achado, é importante salientar, ainda, os arranjos domiciliares e o papel que a mulher exerce na família; considerada tradicional cuidadora dos dependentes, as idosas podem estar mais restritas ao domicílio tanto para cuidar de maridos idosos, quanto para cuidar dos netos 16 .

Ademais, devemos considerar que, entre as idosas longevas, existia um grande percentual de viúvas. Observando-se que a faixa etária em questão recobre uma geração na qual o homem exercia prioritariamente as atividades fora de casa, cabe levantar a hipótese de que esses fatores culturais também possam interferir de alguma forma no desfecho. O estado de viuvez pode ser um fator de influência negativa em relação à capacidade funcional do idoso, o que 
Frequência das variáveis demográficas e de saúde com significância estatística, quando relacionada a sair de casa.

\begin{tabular}{|c|c|c|c|}
\hline \multirow[t]{2}{*}{ Variáveis } & \multirow[t]{2}{*}{ Frequência (\%) } & \multicolumn{2}{|c|}{ Sai de casa (\%) } \\
\hline & & Não & Sim \\
\hline \multicolumn{4}{|l|}{ Faixa etária (anos) } \\
\hline $60-69$ & $3.318(56,3)$ & 12,2 & 87,9 \\
\hline $70-79$ & $1.950(33,1)$ & 22,2 & 77,9 \\
\hline $80+$ & $630(10,7)$ & 35,7 & 64,3 \\
\hline \multicolumn{4}{|l|}{ Sexo } \\
\hline Feminino & $3.064(51,9)$ & 20,2 & 79,8 \\
\hline Masculino & $2.834(48,1)$ & 15,5 & 84,5 \\
\hline \multicolumn{4}{|l|}{ Estado civil } \\
\hline Casado & $2.932(49,7)$ & 14,9 & 85,1 \\
\hline Solteiro & $1.170(19,8)$ & 20,9 & 79,2 \\
\hline Viúvo & $1.796(30,5)$ & 21,1 & 78,9 \\
\hline \multicolumn{4}{|l|}{ Atividades básicas } \\
\hline Baixo & $2.133(36,2)$ & 22,0 & 78,0 \\
\hline Médio & $2.334(39,6)$ & 16,3 & 83,7 \\
\hline Alto & $1.431(24,3)$ & 14,6 & 85,4 \\
\hline \multicolumn{4}{|l|}{ Atividades específicas } \\
\hline Baixo & $2.091(35,5)$ & 23,6 & 76,3 \\
\hline Médio & $1.983(33,6)$ & 15,9 & 84,1 \\
\hline Alto & $1.824(30,9)$ & 13,7 & 86,3 \\
\hline \multicolumn{4}{|l|}{ Queda } \\
\hline Não & $5.410(91,9)$ & 17,3 & 82,8 \\
\hline Sim & $479(8,1)$ & 25,9 & 74,1 \\
\hline \multicolumn{4}{|l|}{ Autopercepção de saúde } \\
\hline Boa & $4.331(73,4)$ & 15,7 & 84,3 \\
\hline Regular & $1.311(22,2)$ & 23,9 & 76,1 \\
\hline Ruim & $256(4,3)$ & 26,2 & 73,8 \\
\hline \multicolumn{4}{|l|}{ Comorbidades } \\
\hline Incontinência urinária & $149(2,5)$ & 24,8 & 75,2 \\
\hline Artrose & $756(12,8)$ & 13,9 & 86,1 \\
\hline Osteoporose & $635(10,8)$ & 19,7 & 80,3 \\
\hline Desnutrição & $16(0,27)$ & 18,8 & 81,3 \\
\hline Cardiopatias & $904(15,3)$ & 22,9 & 77,1 \\
\hline
\end{tabular}

Fonte: Perfil dos Idosos do RS (Instituto de Geriatria e Gerontologia da Pontifícia Universidade Católica do Rio Grande do Sul/ Escola de Saúde Pública do Rio Grande do Sul).

tende a levá-lo a se manter mais restrito em seu domicílio 17.

Como podemos observar entre nossos achados, os idosos casados tendem a sair mais de casa do que os viúvos e solteiros. Acreditamos que os casados sentem-se mais motivados a manter atividades fora de casa e a cuidar da sua saúde pelo fato de terem um companheiro. Em um estudo, o estado civil casado foi relacionado com melhor saúde entre os indivíduos 18. A saúde, por sua vez, está relacionada ao fato de as pessoas saírem mais de casa ou não 19 .
Em relação ao desempenho das atividades básicas e específicas, constatamos que os idosos que as realizam com maior facilidade tendem a sair mais de casa. Na análise ajustada, porém, nota-se que apenas as atividades específicas, as quais exigem maior capacidade funcional, influenciaram o desfecho, indicando que o desenvolvimento das atividades básicas, realizadas especialmente no domicílio, não são determinantes para o idoso sair de casa. Estas, por sua vez, são dependentes das atividades específicas no caso da análise ajustada. Entretanto, de acordo 
Tabela 2

Modelo de regressão logística - análise simples do desfecho "sair de casa".

\begin{tabular}{|c|c|c|c|}
\hline Variáveis independentes & $\mathrm{RC}$ & IC95\% & Valor de $p$ \\
\hline Sexo feminino & 1,00 & & \\
\hline Sexo masculino & 1,38 & $1,21-1,58$ & $<0,001$ \\
\hline Faixa etária 60-69 & 1,00 & & \\
\hline Faixa etária 70-79 & 0,49 & $0,42-0,56$ & $<0,001$ \\
\hline Faixa etária 80 + & 0,25 & $0,21-0,30$ & $<0,001$ \\
\hline Estado civil casado & 1,00 & & \\
\hline Estado civil solteiro & 0,65 & $0,56-0,79$ & $<0,001$ \\
\hline Estado civil viúvo & 0,66 & $0,56-0,76$ & $<0,001$ \\
\hline Autopercepção de saúde boa & 1,00 & & \\
\hline Autopercepção de saúde regular & 0,60 & $0,51-0,69$ & $<0,001$ \\
\hline Autopercepção de saúde ruim & 0,53 & $0,39-0,70$ & $<0,001$ \\
\hline Queda & 0,60 & $0,48-0,75$ & $<0,001$ \\
\hline Incontinência urinária não & 1,00 & & \\
\hline Incontinência urinária NSR * & 1,04 & $0,82-1,32$ & 0,7444 \\
\hline Incontinência urinária Sim & 0,61 & $0,44-0,83$ & 0,0019 \\
\hline Osteoporose & 0,81 & $0,67-0,97$ & 0,0255 \\
\hline Artrose & 1,30 & $1,06-1,60$ & 0,0109 \\
\hline Cardiopatia & 0,83 & $0,70-0,98$ & 0,0311 \\
\hline Desnutrição & 3,19 & $0,76-13,38$ & 0,1128 \\
\hline Atividades básicas baixa & 1,00 & & \\
\hline Atividades básicas média & 1,45 & $1,25-1,68$ & $<0,001$ \\
\hline Atividades básicas alta & 1,65 & $1,38-1,98$ & $<0,001$ \\
\hline Atividades específicas baixa & 1,00 & & \\
\hline Atividades específicas média & 1,63 & $1,40-1,91$ & $<0,001$ \\
\hline Atividades específicas alta & 1,95 & $1,65-2,30$ & $<0,001$ \\
\hline
\end{tabular}

IC95\%: intervalo de 95\% de confiança; RC: razão de chances.

* NSR: não soube responder.

Fonte: Perfil dos Idosos do RS (Instituto de Geriatria e Gerontologia da Pontifícia Universidade Católica do Rio Grande do Sul/ Escola de Saúde Pública do Rio Grande do Sul).

com a literatura, outros fatores podem ter maior associação com o fato de sair de casa do que a dificuldade em realizar as atividades funcionais. Achados sugerem que o declínio funcional não é determinante para restringir o idoso a sua residência, pois, dependendo dos serviços de saúde que recebam, eles podem melhorar a sua capacidade funcional 20,21. Ao mesmo tempo, um estudo com idosos restritos ao lar apontou que $61 \%$ dos participantes apresentavam dificuldades em realizar as ABVDs, enquanto 95\% reportaram dificuldades de mobilidade, especialmente para subir ou descer escadas e caminhar fora de casa, indicando que a saúde física e/ou emocional interferia muito nas atividades sociais 21 . É importante salientar que, devido ao delineamento transversal do presente estudo, não temos como estabelecer a sequência cronológica entre as variáveis independentes e o desfecho; portanto, neste caso, as limitações funcionais podem estar impedindo os idosos de sair de suas casas, ou o fato de eles saírem menos de casa pode estar influenciando negativamente na sua funcionalidade.

Em relação às variáveis relacionadas à saúde dos participantes, a maioria dos idosos referiu ter uma boa autopercepção de saúde. Todavia, idosos que referiram ter saúde regular e ruim saem menos de casa. Um estudo que avaliou os determinantes da autopercepção de saúde de idosos residentes de São Paulo apontou que indivíduos com "uma doença crônica" e "quatro ou mais doenças crônicas” apresentam risco 1,86 e 11,98 vezes maior, respectivamente, de perceber sua saúde ruim, quando comparados àqueles que não apresentam doenças 22 . Os fatores associados à 
Modelo final de regressão logística - análise ajustada do desfecho "sair de casa".

\begin{tabular}{|c|c|c|c|}
\hline Variáveis independentes & $\mathrm{RC}$ & IC95\% & Valor de $p$ \\
\hline Sexo feminino & 1,00 & & \\
\hline Sexo masculino & 1,46 & $1,26-1,68$ & $<0,001$ \\
\hline Faixa etária 60-69 & 1,00 & & \\
\hline Faixa etária 70-79 & 0,52 & $0,44-0,61$ & $<0,001$ \\
\hline Faixa etária 80 + & 0,26 & $0,21-0,33$ & $<0,001$ \\
\hline Estado civil casado & 1,00 & & \\
\hline Estado civil solteiro & 0,70 & $0,59-0,84$ & $<0,001$ \\
\hline Estado civil viúvo & 0,98 & $0,83-1,16$ & 0,8115 \\
\hline Autopercepção de saúde boa & 1,00 & & \\
\hline Autopercepção de saúde regular & 0,71 & $0,61-0,84$ & $<0,001$ \\
\hline Autopercepção de saúde ruim & 0,62 & $0,46-0,84$ & 0,0023 \\
\hline Cardiopatias & 0,77 & $0,65-0,93$ & 0,0058 \\
\hline Artrose & 1,68 & $1,33-2,12$ & $<0,001$ \\
\hline Atividades específicas baixa & 1,00 & & \\
\hline Atividades específicas média & 1,40 & $1,16-1,69$ & 0,0005 \\
\hline Atividades específicas alta & 1,47 & $1,17-1,85$ & 0,0009 \\
\hline
\end{tabular}

IC95\%: intervalo de 95\% de confiança; RC: razão de chances.

Fonte: Perfil dos Idosos do RS (Instituto de Geriatria e Gerontologia da Pontifícia Universidade Católica do Rio Grande do Sul/ Escola de Saúde Pública do Rio Grande do Sul).

autopercepção de saúde estão relacionados com a mortalidade, morbidade e o padrão de cuidado que os idosos exercem sobre sua própria saúde 23 . A autopercepção de saúde como regular ou ruim, somada aos fatores que limitam idosos na presença de doenças crônicas, pode influenciar a escolha por saírem ou não de suas residências, afetando diretamente o convívio social desse contingente populacional.

Das comorbidades analisadas em relação ao desfecho, encontrou-se que idosos com artrose tendem a sair mais de suas residências. Alguns autores enfatizam que a maioria dos idosos vive com pelo menos uma doença crônica não transmissível e que estas, quando controladas, não interferem nas suas atividades rotineiras 24 . Cabe ressaltar que idosos que já apresentam doenças crônicas e recebem acompanhamento para tais tendem a ser mais estimulados pela própria equipe de saúde, com o intuito de se manterem ativos, ocasionando um possível viés de detecção. Os idosos pesquisados estavam aparentemente estáveis clinicamente, pois as doenças osteoarticulares, quando sintomáticas, limitam suas atividades em seu meio de convívio. Sintomas como dor e rigidez articular característicos de artrose podem interferir na qualidade de vida de idosos 25 . Já aqueles com cardiopatias tendem a ficar mais restritos ao domicílio, visto que essas doenças atuam de forma direta na condição clínica do idoso e representam maior causa de mortalidade e morbidade nesse grupo populacional 26. Essa patologia, em face de sua cronicidade, exige certa dependência de medicamentos e serviços de saúde, o que pode levar a uma autopercepção negativa em relação à saúde 27.

Ainda considerando as morbidades analisadas, apenas 3,5\% dos avaliados referiram ser incontinentes. Mesmo que na análise ajustada a incontinência urinária tenha perdido significância estatística na correlação com sair de casa, pesquisas realizadas com pacientes incontinentes, as quais buscavam avaliar a qualidade de vida desses indivíduos, apontaram que a restrição social ocorre devido ao medo ou à vergonha de perderem urina em público, de ficarem molhados e com odor de urina, além do receio de terem dificuldades em encontrar e/ou utilizar o banheiro 28,29. Essa morbidade provoca mudanças no estilo de vida do idoso, que incluem o afastamento de festas, casas de amigos e familiares e até mesmo impedem visitas em sua própria casa ${ }^{11}$; dificulta, ainda, a realização de suas atividades básicas de vida diária em função da perda de urina frequente 6 .

Algumas limitações do estudo devem ser consideradas. Primeiramente, o delineamento transversal, que não permite estabelecer relação 
de causalidade entre as variáveis. A condição de autorrelato das variáveis pode ser considerada um fator limitante, já que as respostas podem ser mais ou menos estimadas pelos participantes, como, por exemplo, na análise da realização das ABVDs e atividades específicas. Outra limitação resultante desses dados é que o instrumento de avaliação das atividades ainda não passou por validação e avaliação das suas propriedades psicométricas. Por outro lado, a condição de autorrelato também pode ser influenciada por déficits cognitivos, de atenção ou depressão. Para evitar esse viés, pacientes que não conseguiram lembrar-se de duas ou mais palavras no teste de recordação foram excluídos. Além disso, como esta é uma análise secundária de dados, muitos fatores clínicos importantes relacionados a sair de casa não foram investigados, como as doenças pulmonares.

\section{Considerações finais}

Ao realizarmos a presente pesquisa, observamos certa dificuldade em encontrar artigos nacionais sobre o desfecho "sair de casa". Considerandose a importância da vida social na qualidade de vida, esperávamos encontrar mais estudos que pudessem subsidiar os nossos achados. Entre os fatores independentes que favoreceram o desfecho em questão, encontramos características sociodemográficas, como ser do sexo masculino, ser de faixa etária mais jovem e ser casado, e de saúde, destacando a presença de artrose e a facilidade em realizar atividades específicas que exigem maior capacidade funcional, além da boa autopercepção de saúde. Entre os fatores negativos, observamos a presença de cardiopatias. Uma vez que, na política de envelhecimento ativo proposta pela OMS 30 , a participação social é um dos pilares norteadores, é extremamente importante considerar condições clínicas que permitem aos idosos manterem-se ativos em comunidade, adequando-se as políticas públicas.

Ressaltamos que nossos achados são relativos a uma pesquisa transversal, que não permite a interpretação causal. Dessa forma, propõe-se a realização de estudos longitudinais, observandose os fatores relacionados com o fato de o idoso deixar de sair de casa. Fatores ambientais também poderiam ser incluídos nos modelos de análise. Em adição, é importante observar os motivos que levam os idosos a saírem de casa ou impedem essa ação. Com base nesses dados, seria possível traçar políticas públicas que visassem a estimular a participação mais ativa do idoso na sociedade, dentro do conceito contemporâneo de envelhecimento ativo 30 .

\section{Resumen}

El estudio tuvo como objetivo evaluar factores (de salud y sociales) involucrados en el hecho de que las personas mayores (60 años o más) salgan de casa. Se encuestaron a 5.898 ancianos, identificados mediante visitas domiciliarias, seleccionados al azar en 59 ciudades del estado de Río Grande do Sul, Brasil. La asociación entre el desenlace y las variables independientes fue analizada mediante regresión logística. Los factores que favorecieron la salida de casa fueron: sexo masculino, edad más joven, estar casado, historia de osteoartritis, facilidad en realizar actividades específicas y buena percepción de salud. La presencia de enfermedades cardiacas fue un factor negativo para salir de casa. Conociendo la importancia de la vida social en la calidad de vida, y en la política de envejecimiento activo propuesta por la Oraganización Mundial de la Salud, es muy importante tener en cuenta las condiciones clínicas que permiten a los ancianos mantenerse activos en la comunidad; estudios como éste pueden ayudar en la adaptación de las políticas públicas para las personas mayores, trabajando principalmente en condiciones cambiantes, tales como la clínica y funcional.

Anciano; Geriatría; Participación Social 


\section{Colaboradores}

P. Morsch contribuiu com a análise dos dados, redação, revisão crítica do manuscrito e aprovação final da versão a ser publicada. G. N. Pereira, J. H. N. Navarro, M. D. Trevisan e D. G. C. Lopes contribuíram com a estruturação do artigo, redação e revisão crítica do manuscrito. Â. J. G. Bós contribuiu com a coleta e análise dos dados, orientação geral e aprovação final da versão a ser publicada.

\section{Referências}

1. Ramos MP, Arend SC. O impacto da reforma da previdência social rural brasileira nos arranjos familiares: uma análise para entender a composição dos domicílios dado o aumento da renda dos idosos. Rev Bras Estud Popul 2012; 29:67-86.

2. Instituto Brasileiro de Geografia e Estatística. Anuário estatístico. http://biblioteca.ibge.gov.br/visu alizacao/periodicos/20/aeb_2000.pdf (acessado em 09/Fev/2014).

3. Souza LM, Lautert L. Trabalho voluntário: uma alternativa para a promoção da saúde em idosos. Rev Esc Enferm USP 2008; 42:371-6.

4. Paschoal SMP. Qualidade de vida na velhice. In: Freitas EV, Py L, organizadores. Tratado de geriatria e gerontologia. Rio de Janeiro: Editora Guanabara Koogan; 2011. p. 99-106.

5. Beck RA, Arizmendi A, Purnell C, Fultz BA, Callahan CM. House calls for seniors: building and sustaining a model of care for homebound seniors. J Am Geriatr Soc 2009; 57:1103-9.

6. Ursine PGS, Cordeiro HA, Moraes CL. Prevalência de idosos restritos ao domicílio em região metropolitana de Belo Horizonte (Minas Gerais, Brasil). Ciênc Saúde Coletiva 2011; 16:2953-62.

7. Organização Mundial da Saúde. Guia global: cidade amiga do idoso. http://www.who.int/ ageing/GuiaAFCPortuguese.pdf (acessado em 27/ Jun/2012).

8. Conselho Estadual do Idoso. Os idosos do Rio Grande do Sul: estudo multidimensional de suas condições de vida: relatório de pesquisa. Porto Alegre: Conselho Estadual do Idoso; 1997.

\section{Agradecimentos}

O presente estudo foi financiado pela Secretaria Estadual de Saúde do Rio Grande do Sul, sendo este trabalho realizado através de parceria entre o IGG/PUCRS e ESP/RS.
9. Pereira GN, Morsch P, Lopes DGC, Trevisan MD, Ribeiro A, Navarro JHN, et al. Fatores socioambientais associados à ocorrência de quedas em idosos. Ciênc Saúde Coletiva 2013; 18:3507-14.

10. Shaw P. Incontinência urinária. Med Update 2003; $1: 8-12$.

11. Honório MO, Santos SMO. Incontinência urinária e envelhecimento: impacto no cotidiano e na qualidade de vida. Rev Bras Enferm 2009; 62:51-6.

12. Romero DE. Diferenciais de gênero no impacto do arranjo familiar no status de saúde dos idosos brasileiros. Ciênc Saúde Coletiva 2002; 7:777-94.

13. Fiedler MM, Peres KG. Capacidade funcional e fatores associados em idosos do Sul do Brasil: um estudo de base populacional. Cad Saúde Pública 2008; 24:409-15.

14. Paskulin LMG, Vianna LAC. Perfil sociodemográfico e condições de saúde auto-referidas de idosos de Porto Alegre. Rev Saúde Pública 2007; 41: 757-68.

15. Pires ZRS, Silva MJ. Autonomia e capacidade decisória dos idosos de baixa renda: uma problemática a ser considerada na saúde do idoso. Rev Eletrônica Enferm 2001; 3(2). http://www.revistas.ufg.br/ index.php/fen/article/view/717/777 (acessado em 01/Fev/2014)

16. Camarano AA, Kanso S, Mello JL, Pasinatto MT. Famílias: espaço de compartilhamento de recursos e vulnerabilidades. In: Camarano AA, organizador. Os novos idosos brasileiros: muito além dos 60 ? Rio de Janeiro: Instituto de Pesquisa Econômica Aplicada; 2004. p. 137-67. 
17. Rosa TEC, Benício MHD, Latorre MRDO, Ramos LR. Fatores determinantes da capacidade funcional entre idosos. Rev Saúde Pública 2003; 37:40-8.

18. Ramos MP. Apoio social e saúde entre idoso. Sociologias 2002; 4:156-75.

19. Cohen-Mansfield J, Shmotkin D, Hazan H. Homebound older persons: prevalence, characteristics, and longitudinal predictors. Arch Gerontol Geriatr 2012; 54:55-60.

20. Gill TM, Williams CS, Richardson ED, Berkman LF, Tinetti ME. A predictive model for ADL dependence in community-living older adults based on a reduced set of cognitive status items. J Am Geriatr Soc 1997; 45:441-5.

21. Charlson ME, Peterson JC, Syat BL, Briggs WM, Kline R, Dodd M, et al. Outcomes of communitybased social service interventions in homebound elders. Int J Geriatr Psychiatry 2008; 23:427-32.

22. Alves LS, Rodrigues RN. Determinantes da auto-percepção de saúde entre idosos do Município de São Paulo, Brasil. Rev Panam Salud Pública 2005; 17:333-41.

23. Silva TR, Menezes PR. Auto-percepção de saúde: um estudo com idosos de baixa renda de São Paulo. Rev Med (São Paulo) 2007; 86:28-38.
24. Rodrigues RAP, Scudeller PG, Pedrazzi EC, Schiavetto FV, Lange C. Morbidade e sua interferência na capacidade funcional de idosos. Acta Paul Enferm 2008; 21:643-8.

25. Franco LR, Simão LS, Pires EO, Guimarães EA. Influência da idade e da obesidade no diagnóstico sugestivo de artrose de joelho. ConScientiae Saúde 2009; 8:41-6.

26. Zaslavsky C, Gus I. Idoso: doença cardíaca e comorbidades. Arq Bras Cardiol 2002; 79:635-9.

27. Jóia LC, Ruiz T, Donalísio MR. Grau de satisfação com a saúde entre idosos do Município de Botucatu, Estado de São Paulo, Brasil. Epidemiol Serv Saúde 2008; 17:187-94.

28. Lopes MHBM, Higa R. Restrições causadas pela incontinência urinária à vida da mulher. Rev Esc Enferm USP 2006; 40:34-41.

29. Nilsson M, Lalos A, Lalos O. The impact of female urinary incontinence and urgency on quality of life and partner relationship. Neurourol Urodyn 2009; 28:976-81.

30. Organização Mundial da Saúde. Envelhecimento ativo: uma política de saúde, 2002. http://dtr2001. saude.gov.br/svs/pub/pdfs/envelhecimento_ati vo.pdf (acessado em 25/Mar/2014).

Recebido em 02/Abr/2014

Versão final reapresentada em 20/Nov/2014

Aprovado em 15/Dez/2014 